\title{
Efficacy of conventional valves compared to programmable valves in managing children $\&$ adolescents with hydrocephalus: a 450 valve retrospective study Timothy Hatlen ${ }^{1}$, David Shurtleff*1, Richard Ellenbogen ${ }^{2}$, Anthony Avellino ${ }^{2}$ and Sharon Duguay ${ }^{1}$
}

\author{
Address: ${ }^{1}$ Department of Paediatrics, MS: A - 7938, University of Washington, 1959 Pacific Avenue N E, Seattle Washington, 98195 , USA and \\ ${ }^{2}$ Department of Neurological Surgery, MS: W - 7, University of Washington, Seattle Washington, 98195, USA \\ Email: David Shurtleff* - david.shurtleff@seattlechildrens.org \\ * Corresponding author
}

from 53rd Annual Meeting of the Society for Research into Hydrocephalus and Spina Bifida Belfast, UK. 24-27 June 2009

Published: 27 November 2009

Cerebrospinal Fluid Research 2009, 6(Suppl 2):S9 doi:10.1186/1743-8454-6-S2-S9

This abstract is available from: http://www.cerebrospinalfluidresearch.com/content/6/S2/S9

(c) 2009 Hatlen et al; licensee BioMed Central Ltd.

\section{Background}

During the past 10 years, programmable cerebrospinal fluid (CSF) shunts (PVS) have increasingly replaced conventional non-programmable shunts (NPVS). However, previous studies are inconclusive to the comparative effectiveness of the valve systems for the treatment of patients with hydrocephalus.

\section{Materials and methods}

The authors collected computerized data for all patients subjected to a CSF shunt insertion or revision except those with brain tumours from the Patient Data Management System and the Computer Information System of the Seattle Children's Hospital from 1 January 2000 through 31 December 2008. Data collected included the patient's diagnosis, birth date, details of the operation insertion and components of revisions, valve type and location, type and timing of prophylactic antibiotic administration, time of day of the operation, duration of operation, presence of a trainee, incidence of shunt complications and white blood cell (WBC) and red blood cell counts (RBC) in the CSF obtained at the time of operation. Patients were followed for a minimum of one year post-operatively and statistical analysis was done using Vassar STATS ${ }^{\varpi}$ and MedCalc ${ }^{\circledast}$ which included Chi Square, Kaplan Meier Survival Curves and multi-variant analysis.

\section{Results}

A total of 1,278 operations were analyzed that incorporated 450 valve (233 PVS \& 217 NPVS) implantations (166) or revisions (284) for 306 patients. Patients with a PVS that were adjusted ( $\geq 6$ mo after implantation) demonstrated no significant difference to rate of non-valve revision $\left(X^{2} p=0.63\right)$, infection $\left(X^{2} p=0.99\right)$, or overall valve survival $(\mathrm{KM} p=0.24)$ compared to patients with NPVS. Complications leading to valve replacement of the initial implantation occurred in $42 \%$ of NPVS and $45 \%$ of $\operatorname{PVS}\left(X^{2} p=0.89\right)$. By differentiating patients by their preoperative WBC (poly $\geq 3$ or mono $\geq 50$ ) and/or RBC $\geq 30$ in the CSF (as indications for inflammation), revisions of NPVS were significantly greater in valve survival rate than the PVS $(p=0.002)$. No significant differences existed between the two valve systems for reason of revision, location, age of patient, time of day, duration of the operation type of antibiotic used or time of administration or presence of trainee.

\section{Conclusion}

This study provides a new rational based upon using preoperative WBC and RBC counts in the CSF to compare the effectiveness between a PVS and a NPVS. As there is a greater complexity involved with a PVS, it is likely that the chance of occlusion of the valve increases which leads to a lower rate of survival compared to the NPVS. 